\title{
Robotic prostatectomy is associated with increased patient travel and treatment delay
}

\author{
Matthew J. Maurice, MD; ${ }^{1}$ Hui Zhu, MD, ${ }^{1,2}$ Simon P. Kim, MD, Robert Abouassaly, MD \\ 'Department of Urology, Glickman Urological and Kidney Institute, Cleveland Clinic, Cleveland, OH, United States; 2 Division of Urology, Louis Stokes Cleveland Veterans Affairs Medical Center, Cleveland, \\ $\mathrm{OH}$, United States; ${ }^{3}$ Urology Institute, University Hospitals Case Medical Center, Cleveland, OH, United States
}

Cite as: Can Urol Assoc J 2016;10(5-6):192-201. htrp://dx.doi.org/10.5489/cuai.3628

See related commentary on page 202.

\section{Abstract}

Introduction: New technologies may limit access to treatment. We investigated radical prostatectomy $(\mathrm{RP})$ access over time since robotic introduction and the impact of robotic use on RP access relative to other approaches in the modern era.

Methods: Using the National Cancer Data Base, RPs performed during the eras of early (2004-2005) and late (2010-2011) robotic dissemination were identified. The primary endpoints, patient travel distance and treatment delay, were compared by era, and for 2010-2011, by surgical approach. Analyses included multivariable and multinomial logistic regression.

Results: 138476 cases were identified, 32\% from 2004-2005 and $68 \%$ from 2010-2011. In 2010-2011, 74\%, 21\%, and 4.3\% of RPs were robotic, open, and laparoscopic, respectively. Treatment in 2010-2011 and robotic approach were independently associated with increased patient travel distance and longer treatment delay $(p<0.001)$. Men treated robotically had 1.1-1.2 times higher odds of traveling medium-to-long-range distances and 1.2-1.3 higher odds of delays 90 days or greater compared to those treated open $(p<0.001)$. Laparoscopic approach was associated with increased patient travel and treatment delay, but to a lesser extent than the robotic approach $(p<0.001)$. In high-risk patients, treatment delays remained significantly longer for minimally invasive approaches $(p<0.001)$. Other factors associated with the robotic approach included referral from an outside facility, treatment at an academic or high-volume hospital, higher income, and private insurance. Potential limitations include the retrospective observational design and lack of external validation of the primary outcomes.

Conclusions: The robotic approach is associated with increased travel burden and treatment delay, potentially limiting access to surgical care.

\section{Introduction}

Over the last 10 years, robotic-assisted radical prostatectomy (RARP) has become the most widely used surgical approach for prostate cancer (PCa) in the U.S. ${ }^{1,2}$ Rapid adoption of robotic technology has caused increased RP use and centralization at high-volume centres. ${ }^{3-5}$ These practice patterns have raised concerns about RP overuse on a population level and decreased access to care on a local-regional level. During early robotic dissemination, RARP was associated with sociodemographic disparities and increased patient travel distances. ${ }^{6-8}$ In the modern era of widespread use, the influence of RARP on access to care has not been investigated. ${ }^{2}$ We examine how RP access has changed over time since robotic introduction and how robotic use has impacted access relative to other approaches in modern times.

\section{Methods}

\section{National Cancer Data Base (NCDB)}

The NCDB is a joint project of the American Cancer Society and the Commission on Cancer $(\mathrm{CoC})$ of the American College of Surgeons. It is a nationwide, hospital-based cancer registry that includes data from over $1500 \mathrm{CoC}$-accredited hospitals, effectively capturing $70 \%$ of cancers in the U.S.

\section{Study population}

After institutional review board approval, we identified patients with clinically localized PCa who underwent RP from 2004-2005 and 2010-2011, excluding patients managed initially with watchful waiting or active surveillance (AS). We selected these time periods to capture early robotic adoption (2004-2005) and widespread use (2010-2011) and to minimize the impact of AS on treatment delay. 
In 2004-2005, RARP accounted for $<10 \%$ of RPs. ${ }^{7,8}$ In 2010-2011, $<10 \%$ of eligible men underwent AS. ${ }^{9}$ A surgical approach identifier, first coded in 2010, was used to differentiate open radical prostatectomy (ORP), laparoscopic radical prostatectomy (LRP), and RARP. Cases missing data on the timing of treatment ( $5 \%$ of cases per year) were excluded $(n=7667)$. Cases missing data on patient travel distance $(\sim 3 \%$ of cases per year) were excluded $(n=4734)$. For the high-risk PCa subgroup analysis, cases with unknown approach were excluded $(n=1400)$.

\section{Study variables}

Demographic factors included race/ethnicity (White or nonWhite), income level, insurance type (uninsured, social, and private), and county. Annual income quartiles were categorized as low $(<\$ 30000)$, low-middle $(\$ 30000-\$ 35$ 000), middle ( $\$ 35000-\$ 46000)$, and upper-middle ( $>46$ 000) based on 2000 U.S. census data. County was categorized as urban, metropolitan, or rural based on 2003 U.S. Department of Agriculture Research Service data.

Clinical factors included age (in years), Charlson comorbidity index $(\mathrm{CCl})$, and $\mathrm{D}^{\prime}$ Amico risk group. $\mathrm{CCl}$ was categorized as 0 (no comorbidities), 1 , or $>1$. D'Amico risk groups were categorized as low (cT1/T2a, prostate-specific antigen $[\mathrm{PSA}] \leq 10 \mathrm{ng} / \mathrm{ml}$, and Gleason score $\leq 6)$, intermediate (T2b, and/or PSA 10-20 ng/mL, and/or Gleason score 7), and high $(\geq \mathrm{T} 2 \mathrm{c}, \mathrm{PSA}>20 \mathrm{ng} / \mathrm{mL}$, or Gleason score 8-10). A combined age- $\mathrm{CCl}$ variable (age $<70$ and $\mathrm{CCl} 0,1$, or $>1$; age $>70$ and $\mathrm{CCl} 0,1$, or $>1$ ) was created to account for collinearity.

Provider factors included hospital type, surgical volume, region, and referral status. Using CoC classifications, hospitals were categorized as academic, comprehensive community, and other. Hospitals were grouped into tertiles based on total RP volume $(<164,164-402$, and $>402)$. Regions included the Northeast, Midwest, South, and West. ${ }^{10}$ Referral status (no or yes) indicated referral from another hospital for treatment.

\section{Study endpoints}

The primary endpoints were patient travel distance to the treating hospital and treatment delay. Travel distance was estimated using the great-circle distance, an established proxy for travel time, and classified by tertiles: short $(<8.2$ miles), medium (8.2-25 miles), and long ( $>25$ miles). Treatment delay was categorized as $<90,90-180$, or $>180$ days.

\section{Statistical analyses}

RPs in 2010-2011 were compared to RPs in 2004-2005, adjusting for age, $\mathrm{CCl}$, race, income, insurance, county, risk group, hospital type, surgical volume, region, and referral status. For 2010-2011, surgical approaches (RARP vs. LRP vs. ORP) were compared to one another, adjusting for the same covariates. This analysis was repeated in the subgroup of men with high-risk PCa. The Chi-squared, Wilcoxon-MannWhitney, and Kruskal-Wallis tests were used for univariate analyses. Multivariable and multinomial logistic regression analyses were used to adjust for covariates. Statistical tests were performed using $\mathrm{SAS}^{\circledR}$ University Edition (SAS Institute Inc., Cary, NC, U.S.). P values $<0.05$ were considered statistically significant.

\section{Results}

The final cohort included 138476 men with predominantly low- and intermediate-risk PCa, including 42702 (31\%) treated in 2004-2005 and 95774 (69\%) treated in 2010-2011. Most men were middle-aged, White, healthy, privately insured, upper-middle-income metropolitans. Median travel distance was 13.1 (interquartile range [IQR] 5.6-35.6) miles, and median treatment delay was two (IQR 1.2-2.9) months.

On univariate analysis (Table 1), patient travel distance and treatment delay differed significantly between the treatment periods $(p<0.001)$. On multivariable analysis (Table 2 ), travel distance and treatment delay remained significant predictors of prostatectomy in 2010-2011 vs. 2004-2005. The odds of travelling medium or long distances were 1.2 and 1.1 times higher, respectively, for men treated in 2010-2011 (OR 1.17, 95\% Cl 1.13-1.20; OR 1.13, 95\% Cl $1.09-1.18 ; \mathrm{p}<0.001)$. The odds of treatment delay $90-180$ days or greater were 1.3 and 1.4 times higher, respectively, for men treated in 2010-2011 (OR 1.30, 95\% Cl 1.25-1.34; OR $1.35,95 \% \mathrm{Cl} 1.26-1.45 ; \mathrm{p}<0.001)$. Furthermore, the use of RP was up to 1.2-1.5-fold higher at high-volume, non-community hospitals in 2010-2011 compared to 2004$2005(\mathrm{p}<0.001)$.

Other factors independently associated with RP in 2010 2011 vs. 2004-2005 were age, CCl, race, insurance, and D'Amico risk group (Table 2). Specifically, RP was more common among older, comorbid, non-White, and socially insured men in 2010-2011 ( $p<0.001)$. Patients treated with $\mathrm{RP}$ in 2010-2011 also were more likely to have high-risk PCa (OR 1.06, 95\% Cl 1.03-1.10; $\mathrm{p}<0.001)$.

In 2010-2011, 70 096/94 374 (74.3\%) men underwent RARP, 20 159/94 374 (21.4\%) underwent ORP, and (4119/94 374) 4.4\% underwent LRP. On univariate and multivariable analyses (Tables 3 and 4), patient travel distance and treatment delay differed significantly by approach. Compared to ORP, the odds of travelling medium-to-long distances were 1.1-1.2 times higher for RARP (medium vs. short distances, OR 1.11, 95\% Cl 1.06-1.16; long vs. short distances, OR $1.15,95 \% \mathrm{Cl} 1.09-1.22 ; \mathrm{p}<0.001)$ and 1.2 
Maurice et al.

\begin{tabular}{|c|c|c|c|c|c|c|c|}
\hline Variables & \multicolumn{2}{|c|}{$\begin{array}{c}\text { Total } \\
(\mathrm{N}=138 \text { 476) }\end{array}$} & \multicolumn{2}{|c|}{$\begin{array}{c}\mathrm{RP}, 2004-2005 \\
(\mathrm{n}=42 \text { 702) }\end{array}$} & \multicolumn{2}{|c|}{$\begin{array}{c}\mathrm{RP}, 2010-2011 \\
(\mathrm{n}=95 \mathrm{774})\end{array}$} & $p$ value \\
\hline Age (years) & & & & & & & $<0.001$ \\
\hline Median (IQR) & \multirow{2}{*}{\multicolumn{2}{|c|}{$\begin{array}{c}61(56-66) \\
60.9(7.1)\end{array}$}} & \multicolumn{2}{|c|}{$61(56-66)$} & \multicolumn{2}{|c|}{$61(56-66)$} & \\
\hline \multirow[t]{2}{*}{ Mean (SD) } & & & \multicolumn{2}{|c|}{$60.4(7.2)$} & \multicolumn{2}{|c|}{$61.0(7.1)$} & \\
\hline & $\mathbf{n}$ & $\%$ & $\mathbf{n}$ & $\%$ & $\mathbf{n}$ & $\%$ & \\
\hline Charlson score & & & & & & & $<0.001$ \\
\hline 0 & 116049 & 83.8 & 36946 & 86.5 & 79103 & 82.6 & \\
\hline 1 & 19973 & 14.4 & 5148 & 12.1 & 14825 & 15.5 & \\
\hline$>1$ & 2454 & 1.8 & 608 & 1.4 & 1846 & 1.9 & \\
\hline Race & & & & & & & $<0.001$ \\
\hline White & 111284 & 80.4 & 34657 & 81.2 & 76627 & 80.0 & \\
\hline Non-White & 23035 & 16.6 & 6509 & 15.2 & 16526 & 17.3 & \\
\hline Unknown & 4157 & 3.0 & 1536 & 3.6 & 2621 & 2.7 & \\
\hline Income level & & & & & & & $<0.001$ \\
\hline Low & 14391 & 10.4 & 4322 & 10.1 & 10069 & 10.5 & \\
\hline Low-middle & 21464 & 15.5 & 6485 & 15.2 & 14979 & 15.6 & \\
\hline Middle & 36428 & 26.3 & 11083 & 26.0 & 25345 & 26.5 & \\
\hline Upper-middle & 64065 & 46.3 & 20139 & 47.2 & 43926 & 45.9 & \\
\hline Unknown & 2128 & 1.5 & 673 & 1.6 & 1455 & 1.5 & \\
\hline Insurance & & & & & & & $<0.001$ \\
\hline Private & 89171 & 64.4 & 28258 & 66.2 & 60913 & 63.6 & \\
\hline Federal/social & 44976 & 32.5 & 12657 & 29.6 & 32319 & 33.7 & \\
\hline Uninsured & 2074 & 1.5 & 565 & 1.3 & 1509 & 1.6 & \\
\hline Unknown & 2255 & 1.6 & 1222 & 2.9 & 1033 & 1.1 & \\
\hline \multicolumn{8}{|l|}{ County } \\
\hline Urban & 21260 & 15.4 & 6231 & 14.6 & 15029 & 15.7 & $<0.001$ \\
\hline Metropolitan & 111105 & 80.2 & 34545 & 80.9 & 76560 & 79.9 & \\
\hline Rural & 3269 & 2.4 & 975 & 2.3 & 2294 & 2.4 & \\
\hline Unknown & 2842 & 2.1 & 951 & 2.2 & 1891 & 2.0 & \\
\hline D'Amico risk group & & & & & & & $<0.001$ \\
\hline Low & 44813 & 32.4 & 14518 & 34.0 & 30295 & 31.6 & \\
\hline Intermediate & 43356 & 31.3 & 14110 & 33.0 & 29246 & 30.5 & \\
\hline High & 32623 & 23.6 & 10113 & 23.7 & 22510 & 23.5 & \\
\hline Unknown & 17684 & 12.8 & 3961 & 9.3 & 13723 & 14.3 & \\
\hline
\end{tabular}

times higher for LRP (medium vs. short distances, OR 1.15, 95\% $\mathrm{Cl} 1.04-1.26 ; \mathrm{p}<0.001)$. Treatment delays 90 days and longer were 1.2-1.3-fold higher for RARP (90-180 vs. $<90$ days, OR $1.28,95 \% \mathrm{Cl} 1.22-1.34 ;>180$ vs. $<90$ days, OR $1.17,95 \% \mathrm{Cl} 1.06-1.29 ; \mathrm{p}<0.001)$ and 1.2 -fold higher for LRP (90-180 vs. <90 days, OR 1.16, 95\% Cl 1.06-1.26; $\mathrm{p}<0.001)$.

LRP and RARP were $13-27 \%$ less likely to be used to treat high-risk PCa on multivariable analysis $(\mathrm{p}<0.001)$. In order to further characterize the effect of risk group on treatment delay, we performed a subgroup analysis of high-risk PCa stratified by approach (Supplementary Table 1). In high-risk $\mathrm{PCa}$, treatment delays remained significantly longer for LRP and RARP $(\mathrm{p}<0.001)$. High-risk PCa treated with LRP or RARP had 1.2-fold and 1.3-fold higher odds, respectively, of treatment delay $90-180$ days compared to ORP $(p<0.001)$. High-risk PCa managed with LRP or RARP also had 1.5-fold higher odds of being referred for treatment and 1.8-2.5-fold higher odds of treatment at a high-volume centre $(p<0.001)$. Treatment delay $>180$ days and travel burden did not differ significantly by approach for high-risk disease.

Other factors independently associated with RP approach for all-risk PCa were referral status, income, hospital type, surgical volume, county, and region. Patients treated with RARP or LRP were 1.2 times and 1.5 times more likely to be referred from an outside facility for treatment than those 


\begin{tabular}{|c|c|c|c|c|c|c|c|}
\hline \multirow[b]{2}{*}{ Variables } & \multicolumn{2}{|c|}{$\begin{array}{c}\text { Total } \\
(\mathrm{N}=138476)\end{array}$} & \multicolumn{2}{|c|}{$\begin{array}{c}\mathrm{RP}, 2004-2005 \\
(\mathrm{n}=42 \text { 702) }\end{array}$} & \multicolumn{2}{|c|}{$\begin{array}{c}\mathrm{RP}, 2010-2011 \\
(\mathrm{n}=95 \mathrm{774})\end{array}$} & \multirow[t]{2}{*}{$p$ value } \\
\hline & $\mathbf{n}$ & $\%$ & $\mathbf{n}$ & $\%$ & $\mathbf{n}$ & $\%$ & \\
\hline Hospital type & & & & & & & $<0.001$ \\
\hline Academic & 59094 & 42.7 & 18193 & 42.6 & 40901 & 42.7 & \\
\hline Comprehensive & 70814 & 51.1 & 21061 & 49.3 & 49753 & 51.9 & \\
\hline Community & 7739 & 5.6 & 2982 & 7.0 & 4757 & 5.0 & \\
\hline Other & 829 & 0.6 & 466 & 1.1 & 363 & 0.4 & \\
\hline Surgical volume & & & & & & & $<0.001$ \\
\hline Low & 46157 & 33.3 & 15427 & 36.1 & 30730 & 32.1 & \\
\hline Intermediate & 46300 & 33.4 & 12932 & 30.3 & 33368 & 34.8 & \\
\hline High & 46019 & 33.2 & 14343 & 33.6 & 31676 & 33.1 & \\
\hline Hospital region & & & & & & & $<0.001$ \\
\hline Northeast & 28682 & 20.7 & 9357 & 21.9 & 19325 & 20.2 & \\
\hline Midwest & 37387 & 27.0 & 10013 & 23.4 & 27374 & 28.6 & \\
\hline South & 47905 & 34.6 & 14725 & 34.5 & 33180 & 34.6 & \\
\hline West & 24502 & 17.7 & 8607 & 20.2 & 15895 & 16.6 & \\
\hline Referred for treatment & & & & & & & 0.015 \\
\hline No & 72572 & 52.4 & 22587 & 52.9 & 49985 & 52.2 & \\
\hline Yes & 65904 & 47.6 & 20115 & 47.1 & 45789 & 47.8 & \\
\hline Distance travelled & & & & & & & $<0.001$ \\
\hline Short & 46377 & 33.5 & 15188 & 35.6 & 31189 & 32.6 & \\
\hline Medium & 45961 & 33.2 & 13624 & 31.9 & 32337 & 33.8 & \\
\hline Long & 46138 & 33.3 & 13890 & 32.5 & 32248 & 33.7 & \\
\hline Treatment delay & & & & & & & $<0.001$ \\
\hline$<90$ days & 102587 & 74.1 & 33009 & 77.3 & 69578 & 72.6 & \\
\hline 90-180 days & 30677 & 22.2 & 8293 & 19.4 & 22384 & 23.4 & \\
\hline$>180$ days & 5212 & 3.8 & 1400 & 3.3 & 3812 & 4.0 & \\
\hline
\end{tabular}

treated with ORP $(\mathrm{p}<0.001)$. The odds of undergoing LRP or RARP vs. ORP were significantly higher for men within the highest income bracket and insured men $(p<0.001)$. Both LRP and RARP were more common at academic and highervolume hospitals $(p<0.001)$.

\section{Discussion}

In order to assess the impact of widespread robotic adoption on practice patterns over time, we compared RPs performed in 2010-2011, when RARP accounted for the majority of cases, to those performed in 2004-2005, when RARP accounted for less than $10 \%$ of cases. ${ }^{2,7,11}$ Consistent with the literature, RPs occurred increasingly at academic and highvolume hospitals over time. ${ }^{4,6}$ This trend has been attributed to the ongoing centralization of complex cancer surgery at high-volume centres and to further technology-driven centralization at centres invested in robotic technology. ${ }^{4-6,12,13}$

While robot-driven centralization may improve the quality of surgical care overall, it also may pose a significant barrier to care for those without access to robotic centres. In a study of RP practice patterns in three Northeastern states from 2000-2009, Stitzenberg et al observed a link between RP centralization and longer patient travel distances. ${ }^{6}$ Nationally, we observed significantly increased travel distances and treatment delays for men treated in 2010-2011 vs. 2004-2005. Since perioperative transportation costs have been shown to disproportionately impact low-income patients, we suspected that socioeconomic factors also might affect access to care. ${ }^{14}$ Surprisingly, we did not detect a significant difference in RP use over time by income level. In fact, in 2010-2011, RP use was significantly more common among traditionally underserved groups, including non-White minorities, Medicare beneficiaries, uninsured men, and urbanites. ${ }^{15}$ Likewise, the treatment of older, comorbid, and high-risk men was significantly higher in 2010-2011. These findings, while somewhat counterintuitive, may be explained by increased RP volume over time, which increased by $124 \%$ between $2004-2005$ and 2010-2011 in our study, and by increased interest in the surgical treatment of high-risk PC., ${ }^{3,16}$ Increased RP volume appears to have improved access to care for underserved men despite greater travel burden and longer treatment delays. 


\begin{tabular}{|c|c|c|c|}
\hline \multirow[b]{3}{*}{ Variables } & \multirow{2}{*}{\multicolumn{3}{|c|}{ 2010-2011 vs. 2004-2005 }} \\
\hline & & & \\
\hline & OR & $95 \% \mathrm{Cl}$ & $p$ value \\
\hline Age & 1.01 & $1.01-1.01$ & $<0.001$ \\
\hline Charlson score & & & $<0.001$ \\
\hline 0 & 1.00 & (referent) & \\
\hline 1 & 1.27 & $1.22-1.32$ & \\
\hline$>1$ & 1.27 & $1.15-1.41$ & \\
\hline Race & & & $<0.001$ \\
\hline White & 1.00 & (referent) & \\
\hline Non-White & 1.22 & $1.18-1.27$ & \\
\hline Income level & & & 0.231 \\
\hline Low & 1.00 & (referent) & \\
\hline Low-middle & 1.01 & $0.95-1.06$ & \\
\hline Middle & 0.99 & $0.94-1.04$ & \\
\hline Upper-middle & 0.97 & $0.92-1.02$ & \\
\hline Insurance & & & $<0.001$ \\
\hline Private & 1.00 & (referent) & \\
\hline Federal/social & 1.07 & $1.03-1.11$ & \\
\hline Uninsured & 1.19 & $1.06-1.33$ & \\
\hline County & & & $<0.001$ \\
\hline Urban & 1.00 & (referent) & \\
\hline Metropolitan & 0.93 & $0.89-0.97$ & \\
\hline Rural & 0.91 & $0.83-1.00$ & \\
\hline D'Amico risk group & & & $<0.001$ \\
\hline Low & 1.00 & (referent) & \\
\hline Intermediate & 0.96 & $0.93-0.99$ & \\
\hline High & 1.06 & $1.02-1.09$ & \\
\hline
\end{tabular}

To further explore the impact of robotic use on access to care, we investigated RP practice patterns in 2010-2011 stratified by approach. In contrast to prior studies, which used hospital robot ownership as a proxy for robotic use, our study demonstrates the impact of actual robotic use on access to care..$^{4,6}$

By 2010-2011, RARP accounted for 74\% of RPs in the U.S., which is consistent with previously reported estimates. ${ }^{2,5,17,18}$ LRP and RARP were more likely at academic and high-volume centres compared to ORP, likely due to centralization, which has occurred to a much greater extent for RARP and LRP than ORP. ${ }^{5}$ This may explain why LRP and RARP were associated with increased patient travel and why RARP was less likely among rural dwellers. LRP and RARP also were performed less commonly in poor and socially insured men, possibly due to increased perioperative travel burden, which disproportionately limits access to care for the poor. ${ }^{14}$ Altogether, these practice patterns suggest that technology-driven centralization may be limiting treatment access and reinforcing healthcare disparities. . $^{4,8,19}$

Patients managed with LRP or RARP in 2010-2011 were significantly more likely to experience treatment delays com-

\begin{tabular}{|c|c|c|c|}
\hline \multirow[b]{2}{*}{ Variables } & \multicolumn{3}{|c|}{$2010-2011$ vs. $2004-2005$} \\
\hline & OR & $95 \% \mathrm{Cl}$ & $p$ value \\
\hline Hospital type & & & $<0.001$ \\
\hline Academic & 1.00 & (referent) & \\
\hline Comprehensive & 1.07 & $1.04-1.10$ & \\
\hline Community & 0.70 & $0.66-0.75$ & \\
\hline Other & 0.29 & $0.24-0.34$ & \\
\hline Surgical volume & & & $<0.001$ \\
\hline Low & 1.00 & (referent) & \\
\hline Intermediate & 1.20 & $1.16-1.24$ & \\
\hline High & 1.16 & $1.11-1.20$ & \\
\hline Hospital region & & & $<0.001$ \\
\hline Northeast & 1.00 & (referent) & \\
\hline Midwest & 1.41 & $1.35-1.46$ & \\
\hline South & 0.99 & $0.95-1.03$ & \\
\hline West & 0.83 & $0.80-0.87$ & \\
\hline Referred for treatment & & & 0.362 \\
\hline No & 1.00 & (referent) & \\
\hline Yes & 0.99 & $0.96-1.02$ & \\
\hline Distance travelled & & & $<0.001$ \\
\hline Short & 1.00 & (referent) & \\
\hline Medium & 1.18 & $1.14-1.22$ & \\
\hline Long & 1.17 & $1.13-1.22$ & \\
\hline Treatment delay & & & $<0.001$ \\
\hline$<90$ days & & (referent) & \\
\hline 90-180 days & 1.30 & $1.26-1.34$ & \\
\hline$>180$ days & 1.35 & $1.26-1.45$ & \\
\hline
\end{tabular}

pared to patients who underwent ORP. We considered that delays may have been influenced by increasing use of, or at least, increasing time spent on the consideration of AS in men with low-risk PCa. However, based on prior research, we know that only $7.4 \%$ of men with D'Amico low-risk PCa were managed with AS in 2010-2011, and these patients were excluded from our study. ${ }^{9}$ Moreover, to further adjust for AS as a confounder, we performed a subgroup analysis of men with high-risk PCa. Even men with high-risk PCa managed by LRP or RARP were more likely to have their surgeries delayed compared to ORP. Increased referral, which occurred preferentially among men undergoing minimally invasive RP, is one possible explanation for these delays. Although it is very unlikely that treatment delays compromise cancer control for low-risk PCa, for higher-risk disease, delays may have an unfavourable impact on oncologic outcomes. ${ }^{20-22}$ Although the quality of the evidence on the association between treatment delay and oncologic outcomes is weak, treatment delay ideally should not exceed 90 days for men with intermediate- or high-risk disease. ${ }^{23}$

This study has some limitations. Due to its retrospective observational design, this study is susceptible to biases. 
RARP and access to care

\begin{tabular}{|c|c|c|c|c|c|c|c|}
\hline \multirow{2}{*}{ Variables } & \multicolumn{2}{|c|}{ ORP (n=20 159) } & \multicolumn{2}{|c|}{ LRP ( $n=4119)$} & \multicolumn{2}{|c|}{ RARP ( $n=70$ 096) } & \multirow[t]{2}{*}{$p$ value } \\
\hline & & & & & & & \\
\hline Age (years) & & & & & & & $<0.001$ \\
\hline Median (IQR) & \multicolumn{2}{|c|}{$62(57-67)$} & \multicolumn{2}{|c|}{$61(56-66)$} & \multicolumn{2}{|c|}{$61(56-66)$} & \\
\hline \multirow[t]{2}{*}{ Mean (SD) } & \multicolumn{2}{|c|}{$61.4(7.2)$} & \multicolumn{2}{|c|}{$61.0(7.2)$} & \multicolumn{2}{|c|}{$60.9(7.1)$} & \\
\hline & $\mathbf{n}$ & $\%$ & $\mathbf{n}$ & $\%$ & $\mathbf{n}$ & $\%$ & \\
\hline Charlson score & & & & & & & $<0.001$ \\
\hline 0 & 16487 & 81.8 & 3392 & 82.4 & 57978 & 82.7 & \\
\hline 1 & 3205 & 15.9 & 647 & 15.7 & 10842 & 15.5 & \\
\hline$>1$ & 467 & 2.3 & 80 & 1.9 & 1276 & 1.8 & \\
\hline Race & & & & & & & $<0.001$ \\
\hline White & 15911 & 78.9 & 3246 & 78.8 & 56325 & 80.4 & \\
\hline Non-White & 3833 & 19.0 & 761 & 18.5 & 11702 & 16.7 & \\
\hline Unknown & 415 & 2.1 & 112 & 2.7 & 2069 & 3.0 & \\
\hline Income level & & & & & & & $<0.001$ \\
\hline Low & 2502 & 12.4 & 435 & 10.6 & 6971 & 9.9 & \\
\hline Low-middle & 3606 & 17.9 & 630 & 15.3 & 10499 & 15.0 & \\
\hline Middle & 5801 & 28.8 & 1075 & 26.1 & 18074 & 25.8 & \\
\hline Upper-middle & 7905 & 39.2 & 1913 & 46.4 & 33535 & 47.8 & \\
\hline Unknown & 345 & 1.7 & 66 & 1.6 & 1017 & 1.5 & \\
\hline Insurance & & & & & & & $<0.001$ \\
\hline Private & 12069 & 59.9 & 2592 & 62.9 & 45400 & 64.8 & \\
\hline Federal/social & 7279 & 36.1 & 1424 & 34.6 & 23130 & 33.0 & \\
\hline Uninsured & 554 & 2.7 & 57 & 1.4 & 865 & 1.2 & \\
\hline Unknown & 257 & 1.3 & 46 & 1.1 & 701 & 1.0 & \\
\hline \multicolumn{8}{|l|}{ County } \\
\hline Urban & 3603 & 17.9 & 627 & 15.2 & 10537 & 15.0 & $<0.001$ \\
\hline Metropolitan & 15571 & 77.2 & 3340 & 81.1 & 56584 & 80.7 & \\
\hline Rural & 614 & 3.0 & 88 & 2.1 & 1563 & 2.2 & \\
\hline Unknown & 371 & 1.8 & 64 & 1.6 & 1412 & 2.0 & \\
\hline D'Amico risk group & & & & & & & $<0.001$ \\
\hline Low & 5492 & 27.2 & 1239 & 30.1 & 23324 & 33.3 & \\
\hline Intermediate & 5406 & 26.8 & 1260 & 30.6 & 22201 & 31.7 & \\
\hline High & 5575 & 27.7 & 982 & 23.8 & 15416 & 22.0 & \\
\hline Unknown & 3686 & 18.3 & 638 & 15.5 & 9155 & 13.1 & \\
\hline Hospital type & & & & & & & $<0.001$ \\
\hline Academic & 7657 & 38.0 & 2208 & 53.6 & 30625 & 43.7 & \\
\hline Comprehensive & 10016 & 49.7 & 1726 & 41.9 & 37225 & 53.1 & \\
\hline Community & 2301 & 11.4 & 154 & 3.7 & 2102 & 3.0 & \\
\hline Other & 185 & 0.9 & 31 & 0.8 & 144 & 0.2 & \\
\hline Surgical volume & & & & & & & $<0.001$ \\
\hline Low & 9993 & 49.6 & 1263 & 30.7 & 18655 & 26.6 & \\
\hline Intermediate & 5581 & 27.7 & 1291 & 31.3 & 26063 & 37.2 & \\
\hline High & 4585 & 22.7 & 1565 & 38.0 & 25378 & 36.2 & \\
\hline
\end{tabular}


Maurice et al.

\begin{tabular}{|c|c|c|c|c|c|c|c|}
\hline \multirow[b]{2}{*}{ Variables } & \multicolumn{2}{|c|}{ ORP (n=20 159) } & \multicolumn{2}{|c|}{$\operatorname{LRP}(n=4119)$} & \multicolumn{2}{|c|}{ RARP (n=70 096) } & \multirow[t]{2}{*}{$p$ value } \\
\hline & $\mathbf{n}$ & $\%$ & $\mathbf{n}$ & $\%$ & $\mathbf{n}$ & $\%$ & \\
\hline Hospital region & & & & & & & $<0.001$ \\
\hline Northeast & 3775 & 18.7 & 998 & 24.2 & 14291 & 20.4 & \\
\hline Midwest & 5656 & 28.1 & 870 & 21.1 & 20436 & 29.2 & \\
\hline South & 7119 & 35.3 & 1821 & 44.2 & 23820 & 34.0 & \\
\hline West & 3609 & 17.9 & 430 & 10.4 & 11549 & 16.5 & \\
\hline Referred for treatment & & & & & & & 0.015 \\
\hline No & 12149 & 60.3 & 1846 & 44.8 & 35305 & 50.4 & \\
\hline Yes & 8010 & 39.7 & 2273 & 55.2 & 34791 & 49.6 & \\
\hline Distance travelled & & & & & & & $<0.001$ \\
\hline Short & 7601 & 37.7 & 1245 & 30.2 & 21765 & 31.1 & \\
\hline Medium & 6544 & 32.5 & 1441 & 35.0 & 23830 & 34.0 & \\
\hline Long & 6014 & 29.8 & 1433 & 34.8 & 24501 & 35.0 & \\
\hline Treatment delay & & & & & & & $<0.001$ \\
\hline$<90$ days & 15749 & 78.1 & 2978 & 72.3 & 49817 & 71.1 & \\
\hline $90-180$ days & 3717 & 18.4 & 976 & 23.7 & 17381 & 24.8 & \\
\hline$>180$ days & 693 & 3.4 & 165 & 4.0 & 2898 & 4.1 & \\
\hline
\end{tabular}

\begin{tabular}{|c|c|c|c|c|c|}
\hline \multirow[b]{2}{*}{ Variables } & \multicolumn{2}{|c|}{ LRP vs. ORP } & \multicolumn{2}{|c|}{ RARP vs. ORP } & \multirow[b]{2}{*}{$p$ value } \\
\hline & OR & $95 \% \mathrm{Cl}$ & OR & $95 \% \mathrm{Cl}$ & \\
\hline Age & 1.00 & $0.99-1.00$ & 1.00 & $1.00-1.00$ & 0.283 \\
\hline Charlson score & & & & & 0.233 \\
\hline 0 & 1.00 & (referent) & 1.00 & (referent) & \\
\hline 1 & 0.99 & $0.89-1.10$ & 1.00 & $0.95-1.05$ & \\
\hline$>1$ & 0.97 & $0.74-1.26$ & 0.86 & $0.76-0.98$ & \\
\hline Race & & & & & 0.794 \\
\hline White & 1.00 & (referent) & 1.00 & (referent) & \\
\hline Non-White & 0.97 & $0.88-1.08$ & 1.01 & $0.96-1.06$ & \\
\hline Income level & & & & & $<0.001$ \\
\hline Low & 1.00 & (referent) & 1.00 & (referent) & \\
\hline Low-middle & 0.93 & $0.80-1.09$ & 0.94 & $0.87-1.01$ & \\
\hline Middle & 1.06 & $0.92-1.22$ & 0.96 & $0.90-1.03$ & \\
\hline Upper-middle & 1.20 & $1.04-1.38$ & 1.18 & $1.10-1.27$ & \\
\hline Insurance & & & & & $<0.001$ \\
\hline Private & 1.00 & (referent) & 1.00 & (referent) & \\
\hline Federal/social & 1.10 & $1.00-1.21$ & 0.98 & $0.93-1.02$ & \\
\hline Uninsured & 0.50 & $0.36-0.70$ & 0.52 & $0.46-0.60$ & \\
\hline Country & & & & & $<0.001$ \\
\hline Urban & 1.00 & (referent) & 1.00 & (referent) & \\
\hline Metropolitan & 1.04 & $0.91-1.18$ & 1.13 & $1.06-1.20$ & \\
\hline Rural & 0.86 & $0.65-1.14$ & 0.86 & $0.77-0.97$ & \\
\hline D'Amico risk group & & & & & $<0.001$ \\
\hline Low & 1.00 & (referent) & 1.00 & (referent) & \\
\hline Intermediate & 1.04 & $0.95-1.14$ & 0.98 & $0.94-1.03$ & \\
\hline High & 0.87 & $0.79-0.96$ & 0.73 & $0.70-0.76$ & \\
\hline
\end{tabular}




\begin{tabular}{|c|c|c|c|c|c|}
\hline \multirow[b]{2}{*}{ Variables } & \multicolumn{2}{|c|}{ LRP vs. ORP } & \multicolumn{2}{|c|}{ RARP vs. ORP } & \multirow[b]{2}{*}{$p$ value } \\
\hline & OR & $95 \% \mathrm{Cl}$ & OR & $95 \% \mathrm{Cl}$ & \\
\hline Hospital type & & & & & $<0.001$ \\
\hline Academic & 1.00 & (referent) & 1.00 & (referent) & \\
\hline Comprehensive & 0.81 & $0.74-0.89$ & 1.46 & $1.40-1.53$ & \\
\hline Community & 0.38 & $0.31-0.48$ & 0.56 & $0.52-0.61$ & \\
\hline Other & 1.25 & $0.80-1.94$ & 0.43 & $0.33-0.57$ & \\
\hline Surgical volume & & & & & $<0.001$ \\
\hline Low & 1.00 & (referent) & 1.00 & (referent) & \\
\hline Intermediate & 1.45 & $1.31-1.61$ & 2.14 & $2.05-2.24$ & \\
\hline High & 1.78 & $1.59-1.98$ & 2.36 & $2.24-2.49$ & \\
\hline Hospital region & & & & & $<0.001$ \\
\hline Northeast & 1.00 & (referent) & 1.00 & (referent) & \\
\hline Midwest & 0.68 & $0.60-0.76$ & 0.92 & $0.87-0.97$ & \\
\hline South & 1.20 & $1.08-1.33$ & 0.85 & $0.81-0.90$ & \\
\hline West & 0.49 & $0.43-0.57$ & 0.75 & $0.71-0.80$ & \\
\hline Referred for treatment & & & & & $<0.001$ \\
\hline No & 1.00 & (referent) & 1.00 & (referent) & \\
\hline Yes & 1.49 & $1.36-1.62$ & 1.19 & $1.14-1.24$ & \\
\hline Distance travelled & & & & & $<0.001$ \\
\hline Short & 1.00 & (referent) & 1.00 & (referent) & \\
\hline Medium & 1.15 & $1.04-1.26$ & 1.11 & $1.06-1.16$ & \\
\hline Long & 0.98 & $0.87-1.10$ & 1.15 & $1.09-1.22$ & \\
\hline Treatment delay & & & & & $<0.001$ \\
\hline$<90$ days & 1.00 & (referent) & 1.00 & (referent) & \\
\hline 90-180 days & 1.16 & $1.06-1.28$ & 1.28 & $1.22-1.34$ & \\
\hline >180 days & 1.03 & $0.85-1.26$ & 1.17 & $1.06-1.29$ & \\
\hline
\end{tabular}


Maurice et al.

Supplementary Table 1. Multinomial logistic regression analysis of predictors of radical prostatectomy for high-risk disease stratified by approach with ORP as the reference group

\begin{tabular}{|c|c|c|c|c|c|}
\hline \multirow[b]{2}{*}{ Variables } & \multicolumn{2}{|c|}{ LRP vs. ORP } & \multicolumn{2}{|c|}{ RARP vs. ORP } & \multirow[b]{2}{*}{$p$ value } \\
\hline & OR & $95 \% \mathrm{Cl}$ & OR & $95 \% \mathrm{Cl}$ & \\
\hline Age & 0.994 & $0.982-1.006$ & 0.990 & $0.985-0.996$ & 0.003 \\
\hline Charlson score & & & & & 0.332 \\
\hline 0 & 1.000 & (referent) & 1.000 & (referent) & \\
\hline 1 & 0.934 & $0.770-1.132$ & 0.961 & $0.879-1.050$ & \\
\hline$>1$ & 1.139 & $0.740-1.754$ & 0.849 & $0.688-1.048$ & \\
\hline Race & & & & & 0.651 \\
\hline White & 1.000 & (referent) & 1.000 & (referent) & \\
\hline Non-White & 1.068 & $0.888-1.285$ & 0.986 & $0.903-1.076$ & \\
\hline Income level & & & & & $<0.001$ \\
\hline Low & 1.000 & (referent) & 1.000 & (referent) & \\
\hline Low-middle & 1.036 & $0.786-1.367$ & 1.036 & $0.915-1.173$ & \\
\hline Middle & 1.114 & $0.860-1.444$ & 1.018 & $0.905-1.145$ & \\
\hline Upper-middle & 1.289 & $0.993-1.673$ & 1.321 & $1.172-1.489$ & \\
\hline Insurance & & & & & $<0.001$ \\
\hline Private & 1.000 & (referent) & 1.000 & (referent) & \\
\hline Federal/social & 1.006 & $0.843-1.201$ & 1.009 & $0.929-1.096$ & \\
\hline Uninsured & 0.219 & $0.102-0.472$ & 0.429 & $0.341-0.540$ & \\
\hline Country & & & & & 0.013 \\
\hline Urban & 1.000 & (referent) & 1.000 & (referent) & \\
\hline Metropolitan & 1.045 & $0.822-1.329$ & 1.114 & $0.996-1.246$ & \\
\hline Rural & 0.633 & $0.354-1.133$ & 0.769 & $0.618-0.958$ & \\
\hline Hospital type & & & & & $<0.001$ \\
\hline Academic & 1.000 & (referent) & 1.000 & (referent) & \\
\hline Comprehensive & 0.698 & $0.593-0.820$ & 1.269 & $1.174-1.372$ & \\
\hline Community & 0.326 & $0.221-0.480$ & 0.528 & $0.457-0.610$ & \\
\hline Other & 0.972 & $0.372-2.537$ & 0.418 & $0.235-0.742$ & \\
\hline Surgical volume & & & & & $<0.001$ \\
\hline Low & 1.000 & (referent) & 1.000 & (referent) & \\
\hline Intermediate & 1.450 & $1.310-1.606$ & 1.947 & $1.799-2.107$ & \\
\hline High & 1.776 & $1.592-1.981$ & 2.543 & $2.306-2.804$ & \\
\hline Hospital region & & & & & $<0.001$ \\
\hline Northeast & 1.000 & (referent) & 1.000 & (referent) & \\
\hline Midwest & 0.832 & $0.670-1.032$ & 1.043 & $0.941-1.156$ & \\
\hline South & 1.108 & $0.906-1.354$ & 0.881 & $0.796-0.974$ & \\
\hline West & 0.600 & $0.461-0.779$ & 0.765 & $0.681-0.860$ & \\
\hline Referred for treatment & & & & & $<0.001$ \\
\hline No & 1.000 & (referent) & 1.000 & (referent) & \\
\hline Yes & 1.543 & $1.326-1.795$ & 1.462 & $1.362-1.569$ & \\
\hline Distance travelled & & & & & 0.210 \\
\hline Short & 1.000 & (referent) & 1.000 & (referent) & \\
\hline Medium & 1.156 & $0.969-1.378$ & 1.033 & $0.954-1.119$ & \\
\hline Long & 1.144 & $0.920-1.422$ & 1.108 & $0.999-1.229$ & \\
\hline Treatment delay & & & & & $<0.001$ \\
\hline$<90$ days & 1.000 & (referent) & 1.000 & (referent) & \\
\hline $90-180$ days & 1.225 & $1.010-1.486$ & 1.336 & $1.214-1.470$ & \\
\hline$>180$ days & 0.594 & $0.339-1.044$ & 1.039 & $0.837-1.291$ & \\
\hline
\end{tabular}


Second, lack of data on robotic use in 2004-2005 prevented us from directly investigating its effect on RP practice patterns over time. However, to our knowledge, surgical approach data from this period does not exist in any other large registry or population-based database. Third, the outcomes in this study have not been externally validated; therefore, the clinical importance of these findings is unclear.

\section{Conclusion}

RARP is associated with increased patient travel and treatment delay, potentially limiting access to care. The clinical significance of these findings remains to be determined.

Competing interests: The authors declare no competing personal or financial interests.

Acknowledgements: The American College of Surgeons and the Commission on Cancer have not verified and are not responsible for the analytic or statistical methodology employed, or the conclusions drawn from these data by the investigator.

This paper has been peer-reviewed.

\section{References}

1. Parsons JK, Messer K, Palazzi K, et al. Diffusion of surgical innovations, patient safety, and minimally invasive radical prostatectomy. JAMA Surg 2014;149:845-51. http://dx.doi.org/10.1001/jamasurg.2014.31

2. Lowrance WT, Eastham JA, Savage C, et al. Contemporary open and robotic radical prostatectomy practice patterns among urologists in the United States. J Urol 2012;187:2087-92.

3. Makarov DV, Yu JB, Desai RA, et al. The association between diffusion of the surgical robot and radical prostatectomy rates. Med Care 201 1;49:333-9. http://dx.doi.org/10.1097/MLR.0b013e318202adb9

4. Anderson CB, Penson DF, Ni S, et al. Centralization of radical prostatectomy in the United States. J Urol 2013;189:500-6. http://dx.doi.org/10.1016/i.juro.2012.10.012

5. Sammon JD, Karakiewicz PI, Sun M, et al. Robot-assisted versus open radical prostatectomy: The differential effect of regionalization, procedure volume and operative approach. J Urol 2013;189:1289-94. http:// dx.doi.org/10.1016/i.juro.2012.10.028

6. Stitzenberg KB, Wong YN, Nielsen ME, et al. Trends in radical prostatectomy: Centralization, robotics, and access to urologic cancer care. Cancer 2012;118:54-62. http://dx.doi.org/10.1002/cncr.26274
7. Hofer MD, Meeks JJ, Cashy J, et al. Impact of increasing prevalence of minimally invasive prostatectomy on open prostatectomy observed in the national inpatient sample and national surgical quality improvement program. J Endouro/ 2013;27:102-7. http://dx.doi.org/10.1089/end.2012.0315

8. Kim SP, Boorijan SA, Shah ND, et al. Disparities in access to hospitals with robotic surgery for patients with prostate cancer undergoing radical prostatectomy. J Urol 2013;189:514-20. http://dx.doi. org/10.1016/i.juro.2012.09.033

9. Maurice MJ, Abouassaly R, Kim SP, et al. Contemporary nationwide patterns of active surveillance use for prostate cancer. JAMA Intern Med 2015;175:1569-71. http://dx.doi.org/10.1001/jamainternmed.2015.2835

10. Maurice MJ, Zhu H, Abouassaly R. A hospital-based study of initial observation for low-risk prostate cancer and its predictors in the United States. Can Urol Assoc J 2015;9:E193-9. http://dx.doi.org/10.5489/ cuaj. 2606

11. Chang SL, Cipriano LE, Harshman LC, et al. Cost-effectiveness analysis of nephron-sparing options for the management of small renal masses. J Urol 2011;185:1591-7. http://dx.doi.org/10.1016/i. juro.2010.12.100

12. Birkmeyer JD, Siewers AE, Finlayson EV, et al. Hospital volume and surgical mortality in the United States. N Engl J Med 2002;346:1128-37. http://dx.doi.org/10.1056/NEJMsa012337

13. Trinh QD, Biartell A, Freedland SJ, et al. A systematic review of the volume-outcome relationship for radical prostatectomy. Eur Urol 2013;64:786-98. http://dx.doi.org/10.1016/j.eururo.2013.04.012

14. Scott AR, Rush AJ 3rd, Naik AD, et al. Surgical followup costs disproportionately impact low-income patients. J Surg Res 2015;199:32-8. http://dx.doi.org/10.1016/i.jss.2015.04.013

15. Marlow NM, Halpern MT, Pavluck AL, et al. Disparities associated with advanced prostate cancer stage at diagnosis. J Health Care Poor Underserved 2010;21:112-31. http://dx.doi.org/10.1353/hpu.0.0253

16. Briganti A, Joniau $S$, Gontero $P$, et al. Identifying the best candidate for radical prostatectomy among patients with high-risk prostate cancer. Eur Urol 2012;61:584-92. http://dx.doi.org/10.1016/j. eururo.2011.11.043

17. Chang SL, Kibel AS, Brooks JD, et al. The impact of robotic surgery on the surgical management of prostate cancer in the USA. BJU Int 2015;115:929-36. http://dx.doi.org/10.1111/bju.12850

18. Kolata G. Results Unproven, Robot Surgery Wins Converts. New York Times. Feb 14.2010:A1.

19. Onega T, Duell EJ, Shi X, et al. Determinants of NCI Cancer Center attendance in Medicare patients with lung, breast, colorectal, or prostate cancer. J Gen Intern Med 2009;24:205-10. http://dx.doi. org/10.1007/s1 1606-008-0863-y

20. Wilt TJ, Brawer MK, Jones KM, et al. Radical prostatectomy versus observation for localized prostate cancer. N Engl J Med 2012;367:203-13. http://dx.doi.org/10.1056/NEJMoal 113162

21. Abern MR, Aronson WJ, Terris MK, et al. Delayed radical prostatectomy for intermediate-risk prostate cancer is associated with biochemical recurrence: Possible implications for active surveillance from the SEARCH database. Prostate 2013;73:409-17. http://dx.doi.org/10.1002/pros.22582

22. Nguyen PL, Whittington R, Koo $\mathrm{S}$, et al. The impact of a delay in initiating radiation therapy on prostatespecific antigen outcome for patients with clinically localized prostate carcinoma. Cancer 2005; 103:20539. http://dx.doi.org/10.1002/cncr.21050

23. van den Bergh $\mathrm{RC}$, Albertsen $\mathrm{PC}$, Bangma $\mathrm{CH}$, et al. Timing of curative treatment for prostate cancer: A systematic review. Eur Urol 2013;64:204-15. http://dx.doi.org/10.1016/.eururo.2013.02.024

Correspondence: Dr. Robert Abouassaly, Urology Insitute, University Hospitals Case Medical Center, Cleveland, OH, United States; robert.abouassaly@uhhospitals.org 